\title{
Quasinormal modes in the field of a dyon-like dilatonic black hole
}

\author{
A. N. Malybayev ${ }^{1, a}$, K. A. Boshkayev ${ }^{1,2,3,4, b}$, V. D. Ivashchuk ${ }^{5,6, c}$ \\ ${ }^{1}$ Department of Theoretical and Nuclear Physics, Al-Farabi Kazakh National University, Al-Farabi Ave., 71, 050040 Almaty, Kazakhstan \\ ${ }^{2}$ National Nanotechnology Laboratory of Open Type, Al-Farabi Kazakh National University, Al-Farabi Ave., 71, 050040 Almaty, Kazakhstan \\ ${ }^{3}$ Department of Engineering Physics, Satbayev University, 22 Satbayev St., 050013 Almaty, Kazakhstan \\ ${ }^{4}$ Ratbay Myrzakulov Eurasian International Centre for Theoretical Physics, Tauelsizdik Ave 27/1-9, 010000 Nur-Sultan, Kazakhstan \\ ${ }^{5}$ Center for Gravitation and Fundamental Metrology, VNIIMS, 46 Ozyornaya St., Moscow 119361, Russian Federation \\ ${ }^{6}$ Institute of Gravitation and Cosmology, Peoples' Friendship University of Russia (RUDN University), 6 Miklukho-Maklaya St., Moscow 117198, \\ Russian Federation
}

\begin{abstract}
Quasinormal modes of massless test scalar field in the background of gravitational field for a non-extremal dilatonic dyonic black hole are explored. The dyon-like black hole solution is considered in the gravitational $4 d$ model involving two scalar fields and two 2-forms. It is governed by two 2-dimensional dilatonic coupling vectors $\lambda_{i}$ obeying $\lambda_{i}\left(\lambda_{1}+\lambda_{2}\right)>0, i=1,2$. The first law of black hole thermodynamics is given and the Smarr relation is verified. Quasinormal modes for a massless scalar (test) field in the eikonal approximation are obtained and analysed. These modes depend upon a dimensionless parameter $a(0<a \leq 2)$ which is a function of $\lambda_{i}$. For limiting strong $(a=+0)$ and weak $(a=2)$ coupling cases, they coincide with the well-known results for the Schwarzschild and Reissner-Nordström solutions. It is shown that the Hod conjecture, connecting the damping rate and the Hawking temperature, is satisfied for $0<a \leq 1$ and all allowed values of parameters.
\end{abstract}

\section{Introduction}

The recent discovery/detection of gravitational waves [1-3] has strengthen a long-living interest to quasinormal modes (QNMs) [4-13], predicted by Vishveshwara in 1970. The detected gravitational waves were emitted during the final (ringdown) stage of binary black hole mergers. The frequencies of these waves were governed by a certain superpositions of decaying oscillations, i.e. QNMs. The careful analysis of these experiments may be rather important since it can shed some light on nature of gravity in a strong field regime.

\footnotetext{
a e-mail: algis_malybayev@mail.ru

be-mail: kuantay@mail.ru

c e-mail: ivashchuk@mail.ru (corresponding author)
}

From the mathematical point of view, the quasinormal mode (QNM) problem can be reduced to studying the solutions to a wave equation for a scalar function $\Phi(t, x)$ chosen in the following form

$\Phi(t, x)=e^{-i \omega t} \Phi_{*}(x)$,

where $\Phi_{*}=\Phi_{*}(x)$ obeys a Schrödinger-type equation

$$
\left(-\varepsilon^{2} \frac{d^{2}}{d x^{2}}+V(x)\right) \Phi_{*}=\omega^{2} \Phi_{*}
$$

defined on a certain domain of real line $\mathbb{R}=(-\infty,+\infty)$, where $\varepsilon>0$ is some parameter, e.g. $\varepsilon=1$; for reviews see [10-13]. For asymptotically flat black-hole solutions the functions $\Phi_{*}(x)$ are defined on $\mathbb{R}$. In this case $x$ is chosen as a so-called tortoise coordinate (in the body of the paper denoted as $R_{*}$ ), and (at least) for certain known spherically symmetric solutions (e.g. Schwarzschild and Reissner-Nordström ones) the potential $V(x)$ is a positively defined smooth function, having sufficiently fast fall off (to zero) in approaching either to the horizon $(x \rightarrow-\infty)$ or to the spatial infinity $(x \rightarrow$ $+\infty$ ). Usually QNM frequencies $\omega$ are defined as complex numbers obeying $\operatorname{Re} \omega>0$ and $\operatorname{Im} \omega<0$, such that the wave functions (1) are exponentially damped in time as $(t \rightarrow$ $+\infty$ ), corresponding to asymptotically stable perturbations. The QNM frequencies appear for the solutions to Eq. (2) which behave as outgoing waves at spatial infinity: $\Phi_{*}(x) \sim$ $e^{\frac{i \omega x}{\varepsilon}}$ for $x \rightarrow \infty(\operatorname{Re} \omega>0)$ and ingoing ones at the horizon: $\Phi_{*}(x) \sim e^{-\frac{i \omega x}{\varepsilon}}$ for $x \rightarrow-\infty$ with exponential growth (in $|x|$ ) for $\left|\Phi_{*}(x)\right|$ as $|x| \rightarrow \infty$ (due to $\operatorname{Im} \omega<0$ ).

For calculation of QNM $[12,13]$ there exists a (most popular) method, introduced in Refs. [7-9], which may be called as analytical continuation method. The most transparent version of this method was recently proposed (and verified) in Ref. [14]. Here we consider for simplicity the case of the 
potential defined on all real axis $\mathbb{R}=(-\infty,+\infty)$ to avoid the boundary problems. (For more involved and subtle case when the Schrödinger operator and effective potential are defined on $(0,+\infty)$ and proper boundary condition should be specified see Ref. [15].) The prescription is as follows: one should start with the Schrödinger equation for non-relativistic quantum particle (of mass $1 / 2$ ) moving in the inverted potential $-V(x)$ :

$$
\left(-\hbar^{2} \frac{d^{2}}{d x^{2}}-V(x)\right) \Psi=E \Psi
$$

where $\Psi=\Psi(x)$ is the wave function. For a potential under consideration the inverted potential $-V(x)$ may have certain bounded states described by discrete spectrum energy levels $E_{n}=E(\hbar, n \mid-V)$, where $n=0,1, \ldots$ The corresponding wave functions $\Psi=\Psi_{n}(x)$ for a proper potential under consideration is exponentially decaying at both infinities, i.e. $\Psi_{n} \sim e^{\mp \sqrt{-E_{n}} \frac{x}{\hbar}}$ as $x \rightarrow \pm \infty$. The Hatsuda's (analytical continuation) approach [14] tells us that QNM frequencies for the potential $V(x)$ may be obtained from bounded states for the inverted potential $-V(x)$ by putting formally $\hbar=i \varepsilon$ in (2). Hence, due to this prescription we get the following QNM frequencies

$\omega^{2}=-E(\hbar=i \varepsilon, n \mid-V)$,

where $n=0,1, \ldots$ is called as (QNM) overtone number.

It should be noted, that the method suggested in Ref. [14] for computation quasinormal frequencies of spherically symmetric black holes, relates them to bound state energies of anharmonic oscillators by using the analytic continuation in $\hbar$. It was stated in Ref. [14], that the known WKB results are easily reproduced by this method and, moreover, "the perturbative WKB series of the quasinormal frequencies turn out to be Borel summable divergent series both for the Schwarzschild and for the Reissner-Nordström black holes".

In this paper we continue our previous studies [16-18] devoted to dilatonic dyon and dyon-like black hole solutions. Dilatonic and dyon-like black hole solutions were considered in numerous papers, see [19-22] and [23-29], respectively, and references therein. We note that earlier the main motivation for considering the dilaton scalar fields was coming from (super)string theory or certain higher-dimensional (e.g. supergravity) models.

Here we study QNM spectrum in eikonal approximation for a special dyon-like dilatonic black hole solution from Ref. [30], with electric and magnetic color charges $Q_{1}$ and $Q_{2}$, respectively, in the $4 d$ model with metric $g$, two scalar fields $\varphi^{1}, \varphi^{2}$, two 2-forms $F^{(1)}$ and $F^{(2)}$, corresponding to two dilatonic coupling vectors $\lambda_{1}$ and $\lambda_{2}\left(\lambda_{1} \neq-\lambda_{2}\right)$, respectively.

These eikonal QNM modes depend upon a dimensionless parameter $a(0<a \leq 2)$ which is a function of $\lambda_{i}$. It should be noted that QNMs for dilatonic black holes were considered in numerous papers, see Refs. [31-39] and references therein.

The relation between the 2-forms and color charges are given by

$F^{(1)}=Q_{1} \tau_{1}, \quad F^{(2)}=Q_{2} \tau_{2}$,

where $\tau_{2}=\operatorname{vol}\left[S^{2}\right]$ is magnetic 2-form, which is volume form on 2-dimensional sphere and $\tau_{1}$ is an "electric" 2 -form.

We note that in the case of one scalar field $\varphi$ and two coupling constants $\lambda_{1}, \lambda_{2}$ the dyon-like ansatz was considered recently in Refs. $[17,18,28,29]$. For $\lambda_{1}=\lambda_{2}=\lambda$ our solutions from Ref. [17] were dealing with a trivial noncomposite generalization of dilatonic dyon black hole solutions in the model with one 2-form and one scalar field which was considered in Ref. [16], see also Refs. [24-27], and references therein.

The solutions with one scalar field from Refs. [17, 18] may be embedded to the solutions under examination by considering the case of collinear dilatonic coupling vectors:

$\lambda_{1}=\lambda_{1} e, \quad \lambda_{2}=\lambda_{2} e$,

where $e^{2}=1, \lambda_{1}+\lambda_{2} \neq 0$.

The paper is organised as follows: in Sect. 2 we review the main properties of the black hole dyon solution from Ref. [30]. In Sect. 3 we consider the physical parameters and particular cases of the dyonic black hole solutions. In Sect. 4 we analytically derive the eikonal approximation for frequencies of QNM corresponding to massless test scalar field in the background metric of our dyonic-like black hole solution and study their features. In Sect. 5 we consider two limiting cases $a=+0$ and $a=2$, corresponding to the Schwarzschild and Reissner-Nordström black hole solutions. In Sect. 6 we test/check the validity of the Hod conjecture [40] for the solution under consideration when $0<a \leq 2$. Finally, we summarize our conclusion in Sect. 7.

\section{Black hole dyon solutions}

The action of a model containing two scalar fields, 2-form and dilatonic coupling vectors which was considered in Ref. [30], is following

$$
\begin{aligned}
S= & \frac{1}{16 \pi G} \int d^{4} x \sqrt{|g|}\left\{\mathcal{R}[g]-g^{\mu \nu} \partial_{\mu} \varphi \partial_{\nu} \varphi\right. \\
& \left.-\frac{1}{2} e^{2 \lambda_{1} \varphi} F_{\mu \nu}^{(1)} F^{(1) \mu \nu}-\frac{1}{2} e^{2 \lambda_{2} \varphi} F_{\mu \nu}^{(2)} F^{(2) \mu \nu}\right\},
\end{aligned}
$$

where $g=g_{\mu \nu}(x) d x^{\mu} \otimes d x^{\nu}$ is the metric, $|g|=\left|\operatorname{det}\left(g_{\mu \nu}\right)\right|$, $\varphi=\left(\varphi^{1}, \varphi^{2}\right)$ is the vector of scalar fields belonging to $\mathbb{R}^{2}$, $F^{(i)}=d A^{(i)}=\frac{1}{2} F_{\mu \nu}^{(i)} d x^{\mu} \wedge d x^{\nu}$ is the 2-form with $A^{(i)}=$ $A_{\mu}^{(i)} d x^{\mu}, i=1,2, G$ is the gravitational constant, $\lambda_{1}=$ $\left(\lambda_{1 i}\right) \neq 0, \lambda_{2}=\left(\lambda_{2 i}\right) \neq 0$ are the dilatonic coupling vectors 
obeying

$\lambda_{1} \neq-\lambda_{2}$

and $\mathcal{R}[g]$ is the Ricci scalar. Here and in what follows we put $c=1$ (where $c$ is the speed of light in vacuum).

We consider a dyonic-like black hole solution to the field equations corresponding to the action (7) which has the following form [30]

$$
d s^{2}=H^{a}\left\{-H^{-2 a}\left(1-\frac{2 \mu}{R}\right) d t^{2}+\frac{d R^{2}}{1-\frac{2 \mu}{R}}+R^{2} d \Omega^{2}\right\},
$$

$$
\varphi^{i}=v^{i} \ln H,
$$

$F^{(1)}=\frac{Q_{1}}{H^{2} R^{2}} d t \wedge d R, \quad F^{(2)}=Q_{2} \tau$,

where $Q_{1}$ and $Q_{2}$ are (color) charges - electric and magnetic, respectively, $\mu>0$ is the extremality parameter, $d \Omega^{2}=$ $d \theta^{2}+\sin ^{2} \theta d \phi^{2}$ is the canonical metric on the unit sphere $S^{2}(0<\theta<\pi, 0<\phi<2 \pi), \tau=\sin \theta d \theta \wedge d \phi$ is the standard volume form on $S^{2}$,

$H=1+\frac{P}{R}$,

with $P>0$ obeying

$P(P+2 \mu)=\frac{1}{2} Q^{2}$.

All the rest parameters of the solution are defined as follows

$a=\frac{\left(\lambda_{1}+\lambda_{2}\right)^{2}}{\Delta}$,

$\Delta \equiv \frac{1}{2}\left(\lambda_{1}+\lambda_{2}\right)^{2}+\lambda_{1}^{2} \lambda_{2}^{2}-\left(\lambda_{1} \lambda_{2}\right)^{2}$,

$v^{i}=\frac{\lambda_{1 i} \lambda_{2}\left(\lambda_{1}+\lambda_{2}\right)-\lambda_{2 i} \lambda_{1}\left(\lambda_{1}+\lambda_{2}\right)}{\Delta}$,

$i=1,2$ and

$Q_{1}^{2}=\frac{\lambda_{2}\left(\lambda_{1}+\lambda_{2}\right)}{2 \Delta} Q^{2}, \quad Q_{2}^{2}=\frac{\lambda_{1}\left(\lambda_{1}+\lambda_{2}\right)}{2 \Delta} Q^{2}$.

Here the following additional restrictions on dilatonic coupling vectors are imposed

$\lambda_{i}\left(\lambda_{1}+\lambda_{2}\right)>0, \quad i=1,2$.

Correspondingly, we note that

$\Delta>0$,

is valid for $\lambda_{1} \neq-\lambda_{2}$.

Due to relations (18) and (19) the $Q_{s}^{2}$ are well-defined. Note that the restrictions (18) imply relations $\lambda_{s} \neq 0, s=$ 1,2 , and (8).

Indeed, in this case we have the sum of two non-negative terms in (16): $\left(\lambda_{1}+\lambda_{2}\right)^{2}>0$ and

$C=\lambda_{1}^{2} \lambda_{2}^{2}-\left(\lambda_{1} \lambda_{2}\right)^{2} \geq 0$, due to the Cauchy-Schwarz inequality. Moreover, $C=0$ if and only if vectors $\lambda_{1}$ and $\lambda_{2}$ are collinear. Relation (20) implies

$0<a \leq 2$

For non-collinear vectors $\lambda_{1}$ and $\lambda_{2}$ we get $0<a<2$ while $a=2$ for collinear ones.

This solution may be verified just by a straightforward substitution into the equations of motion.

The calculation of scalar curvature for the metric $d s^{2}=$ $g_{\mu \nu} d x^{\mu} d x^{\nu}$ in (9) yields

$\mathcal{R}[g]=\frac{a(2-a) P^{2}(R-2 \mu)}{2 R^{3-a}(R+P)^{2+a}}$.

\section{Particular cases and physical parameters}

Here we analyze certain cases and physical parameters corresponding to the solutions under consideration.

\subsection{Non-collinear and collinear cases}

Non-collinear case For non-collinear vectors $\lambda_{1}$ and $\lambda_{2}(0<$ $a<2$ ) we obtain

$\mathcal{R}[g] \rightarrow-\infty$

as $R \rightarrow+0$ and hence we have a black hole with a horizon at $R=2 \mu$ and singularity at $R=+0$.

Collinear case For collinear vectors $\lambda_{1}, \lambda_{2}$ from (6) obeying $\lambda_{1}+\lambda_{2} \neq 0$ we obtain $v^{i}=0, a=2$ and

$Q_{1}^{2}=\frac{\lambda_{2}}{\lambda_{1}+\lambda_{2}} Q^{2}, \quad Q_{2}^{2}=\frac{\lambda_{1}}{\lambda_{1}+\lambda_{2}} Q^{2}$,

where $\lambda_{1} \lambda_{2}>0$. By changing the radial variable, $R=r-P$, we get a little extension of the solution from Ref. [17]

$d s^{2}=-f(r) d t^{2}+f(r)^{-1} d r^{2}+r^{2} d \Omega_{2}^{2}$,

$F^{(1)}=\frac{Q_{1}}{r^{2}} d t \wedge d r, \quad F^{(2)}=Q_{2} \tau, \quad \varphi=0$,

where $f(r)=1-\frac{2 G M}{r}+\frac{Q^{2}}{2 r^{2}}, Q^{2}=Q_{1}^{2}+Q_{2}^{2}$ and $G M=$ $P+\mu=\sqrt{\mu^{2}+\frac{1}{2} Q^{2}}$.

The metric in these variables coincides with the wellknown Reissner-Nordström metric governed by two parameters: $G M>0$ and $Q^{2}<2(G M)^{2}$. We have two horizons in this case. The electric and magnetic charges are not independent but obey Eqs. (24). Note that to be consistent with the literature the net charge here is related to the charge of the Reissner-Nordström black hole as follows $Q^{2}=2 Q_{R N}^{2}$. 


\subsection{Gravitational mass and scalar charges}

The definition of the ADM gravitational mass is obtained from Eq. (9) in the weak field regime by comparing with $g_{00}=-(1-2 G M / R+O(1 / R))$

$G M=\mu+\frac{a}{2} P$.

In turn, the scalar charge vector $Q_{\varphi}=\left(Q_{\varphi}^{1}, Q_{\varphi}^{2}\right)$ is derived from (10) in the weak field regime using the following definition: $\varphi^{i}=Q_{\varphi}^{i} / R+O(1 / R)$ :

$Q_{\varphi}=\nu P$,

where $v$ is given by Eq. (16).

By combining relations (27) and (28) we obtain the following identity

$2(G M)^{2}+Q_{\varphi}^{2}=Q_{1}^{2}+Q_{2}^{2}+2 \mu^{2}$.

This formula does not contain vectors $\lambda_{s}$.

The identity (29) may be verified by using (14), (17) and the following relation

$v^{2}=\frac{\left(\lambda_{1}+\lambda_{2}\right)^{2}\left(\lambda_{1}^{2} \lambda_{2}^{2}-\left(\lambda_{1} \lambda_{2}\right)^{2}\right)}{\Delta^{2}}=\frac{a(2-a)}{2}$.

For further analyses it is convenient to introduce the following dimensionless parameters

$p=P / \mu>0, \quad q=|Q| /(G M)$.

We obtain

$f_{*}(p, a)=\frac{p(p+2)}{\left(1+\frac{a}{2} p\right)^{2}}=\frac{q^{2}}{2}$.

The function $f_{*}(p, a)$ is monotonically increasing in $p$ on $(0,+\infty)$ for any $a \in(0,2]$ since

$\frac{\partial}{\partial p} f_{*}=\frac{16\left[\left(1-\frac{a}{2} p\right)+1\right]}{(2+a p)^{3}}>0$.

Due to (32) and $\lim _{p \rightarrow+\infty} f_{*}(p, a)=4 / a^{2}$ relation (32) defines a one-to-one correspondence between $p \in(0,+\infty)$ and $q \in\left(0, \frac{2 \sqrt{2}}{a}\right)$ for any (fixed) $a \in(0,2]$. Thus, we have

$0<q^{2}<\frac{8}{a^{2}}, \quad 0<Q^{2}<\frac{8}{a^{2}}(G M)^{2}$

The inverse map $p(q)=p(q, a)$ is defined for any $a \in(0,2]$ as follows

$p=\frac{8 \sqrt{\frac{1}{2}(1-a) q^{2}+1}+2 a q^{2}-8}{8-a^{2} q^{2}}$.

\subsection{Black hole thermodynamics}

In this subsection we consider black hole thermodynamics by calculating the Hawking temperature and entropy, checking the first law (of black hole thermodynamics) and testing the Smarr relation.

To this end, for simplicity here we put $\hbar=c=k_{B}=$ 1. The Bekenstein-Hawking (area) entropy $S=A /(4 G)$, associated with the black solution (12) at the horizon at $R=$ $2 \mu$, where $A$ is the horizon area, reads

$S=S_{B H}=\frac{4 \pi \mu^{2}}{G}\left(1+\frac{P}{2 \mu}\right)^{a}$,

while the related Hawking temperature is following one

$T=T_{H}=\frac{1}{8 \pi \mu}\left(1+\frac{P}{2 \mu}\right)^{-a}$.

It may be verified that relations (27), (36) and (37) imply the first law of the black hole thermodynamics

$d M=T d S+\Phi d Q$

as well as the Smarr formula

$M=2 T S+\Phi Q$

where

$\Phi=\frac{a Q}{4 G(P+2 \mu)}$

Relations (38), (39) may be presented in the following form

$d M=T d S+\Phi_{1} d Q_{1}+\Phi_{2} d Q_{2}$,

$M=2 T S+\Phi_{1} Q_{1}+\Phi_{2} Q_{2}$,

where

$\Phi_{i}=\frac{Q_{i}}{2 G(P+2 \mu)}$,

$i=1,2$. In derivation of relations (41), (42) the following identity is used

$Q_{1}^{2}+Q_{2}^{2}=\frac{a}{2} Q^{2}$

Let us clarify the physical sense of potentials (43). The first relation in (11) for $F^{(1)}=d A^{(1)}$, has a special solution for 1-form:

$A^{(1)}=A_{0}^{(1)}(R) d t=\frac{Q_{1}}{R+P} d t$.

Thus, we get

$\Phi_{1}=\frac{1}{2 G} A_{0}^{(1)}(2 \mu)$

i.e. $\Phi_{1}$ is coinciding up to a factor $1 /(2 G)$ with the value of the zero component of the first Abelian gauge field $A^{(1)}$, or 
electric potential, (in chosen gauge) for the field of electric charge at the horizon.

Now let us consider the magnetic term in (11). The calculation of Hodge-dual gives us

$* F^{(2)}=\frac{Q_{2}}{H^{a} R^{2}} d t \wedge d R$,

(here $\left.* F_{\mu \nu}=\frac{1}{2} \sqrt{|g|} \varepsilon_{\mu \nu \rho \sigma} F^{\rho \sigma}, \varepsilon_{0123}=1\right)$. Relation (16) implies

$\lambda_{2} v=\frac{a}{2}-1$

and hence (see (10))

$e^{2 \lambda_{2} \varphi}=H^{a-2}$.

For $S$-dual 2-form

$\tilde{F}^{(2)}=d \tilde{A}^{(2)}=e^{2 \lambda_{2} \varphi} * F^{(2)}$

we get

$\tilde{F}^{(2)}=\frac{Q_{2}}{H^{2} R^{2}} d t \wedge d R$

and we can choose a corresponding 1-form as follows

$\tilde{A}^{(2)}=\tilde{A}_{0}^{(2)}(R) d t=\frac{Q_{2}}{R+P} d t$.

Hence,

$\Phi_{2}=\frac{1}{2 G} \tilde{A}_{0}^{(2)}(2 \mu)$

i.e. $\Phi_{2}$ is coinciding up to a factor $1 /(2 G)$ with the value of the zero component of the dual Abelian gauge field $\tilde{A}^{(2)}$, or dual electric potential, (in chosen gauge) at the horizon, which corresponds to the field of magnetic charge modulated by scalar fields.

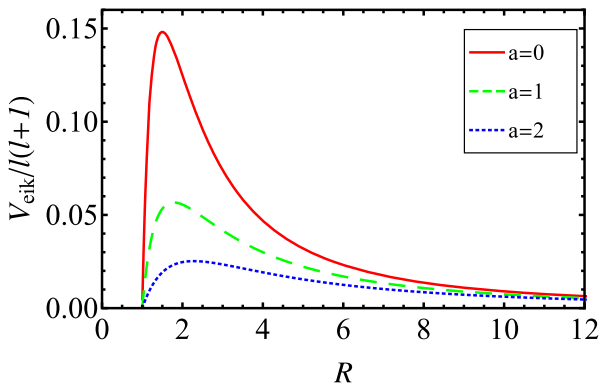

Fig. 1 The eikonal part of the effective potential $V_{e i k}=\mathcal{V}$ for a dyon black hole solution. Left panel: The reduced (eikonal) effective potential $\mathcal{V} / l(l+1)$ as a function of $R$. Right panel: The reduced (eikonal) effective potential $\mathcal{V} / l(l+1)$ as a function of $R_{*}$. In both

\section{Quasinormal modes}

In this section we derive quasinormal modes (in eikonal approximation) for our static and spherically symmetric solution with the metric given (initially) in the following general form

$d s^{2}=-A(u) d t^{2}+B(u) d u^{2}+C(u) d \Omega^{2}$,

where $A(u), B(u), C(u)>0$ and $d \Omega^{2}=d \theta^{2}+\sin ^{2} \theta d \phi^{2}$. Note that in this section and below we use the Planck units, i.e. we put $\hbar=G=c=1$.

We consider a test massless scalar field defined in the background given by the metric (54). The equation of motion in general is written in the form of the covariant Klein-FockGordon equation

$\Delta \Psi=\frac{1}{\sqrt{|g|}} \partial_{\mu}\left(\sqrt{|g|} g^{\mu \nu} \partial_{\mu} \Psi\right)=0$,

where $\mu, v=0,1,2,3$. In order to solve this equation we separate variables in function $\Psi$ as follows

$\Psi=e^{-i \omega t} e^{-\gamma} \Psi_{*}(u) Y_{l m}$,

where $Y_{l m}$ are the spherical harmonics. Equation (55), after using (56) yields the equation describing the radial function $\Psi_{*}(u)$ and having a Schrödinger-like form

$\frac{d^{2} \Psi_{*}(u)}{d u^{2}}+\left\{\frac{B}{A} \omega^{2}-\frac{B}{C} l(l+1)-\gamma^{\prime \prime}-\left(\gamma^{\prime}\right)^{2}\right\} \Psi_{*}(u)=0$

where

$\gamma=\frac{1}{2} \ln \left(B^{-1} C \sqrt{A B}\right)$

and $\gamma^{\prime}=d \gamma / d u$, and $l$ is the multipole quantum number, $l=0,1, \ldots$.

Taking into account above expressions one can examine a dyon-like black hole solution which has the following form

$d s^{2}=-f(R) d t^{2}+\frac{d R^{2}}{f(R)}+H^{a} R^{2} d \Omega^{2}$,

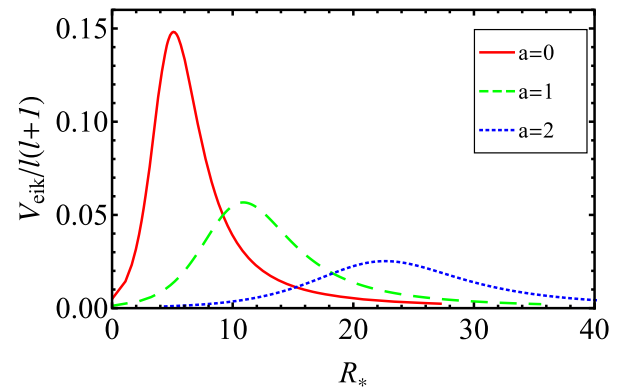

panels the solid red, dashed green and dotted blue curves correspond to $a=0, a=1$ and $a=2$ cases, respectively. For numerical goals here we adopted $P=1$ and $\mu=1 / 2$ 
where $f(R)$ and $C(R)$ according to Eq. (10) can be written as

$$
\begin{aligned}
f(R) & =A=H^{-a}\left(1-\frac{2 \mu}{R}\right), \\
C & =H^{a} R^{2},
\end{aligned}
$$

where $H(R)=1+P / R$ is the moduli function, $\mu, P>0$ and $0<a \leq 2$ as shown earlier. After using the "tortoise" coordinate transformation

$d R_{*}=\frac{d R}{f}$

the metric takes the following form

$d s^{2}=-f d t^{2}+f d R_{*}^{2}+C d \Omega^{2}$.

For the choice of the tortoise coordinate as a radial one ( $u=$ $R_{*}$ ) we have $A=B=f$ and

$\gamma=\frac{1}{2} \ln C=\frac{1}{2} \ln \left(H^{a} R^{2}\right)$.

Thus, the Klein-Fock-Gordon equation becomes

$\frac{d^{2} \Psi_{*}}{d R_{*}^{2}}+\left\{\omega^{2}-V\right\} \Psi_{*}=0$,

where $\omega$ is the (cyclic) frequency of the quasinormal mode and $V=V(R)=V\left(R\left(R_{*}\right)\right)$ is the effective potential

$$
\begin{aligned}
V= & \mathcal{V}+\delta \mathcal{V}, \\
\mathcal{V}= & \frac{l(l+1) f}{H^{a} R^{2}}, \\
\delta \mathcal{V}= & \gamma^{\prime \prime}+\left(\gamma^{\prime}\right)^{2}=\frac{f^{2} a(a-2) P^{2}}{4 H^{2} R^{4}} \\
& +\frac{f(2 R-(a-2) P)(a P(R-2 \mu)+2 \mu(P+R))}{2 H^{a+2} R^{5}},
\end{aligned}
$$

so that $\mathcal{V}$ is the eikonal part of the effective potential. Here and below we denote $F^{\prime}=\frac{d F}{d R_{*}}=f \frac{d F}{d R}$.

In what follows we consider the so-called eikonal approximation when $l \gg 1$.

The maximum of the eikonal part of the effective potential is found from the condition

$$
\begin{aligned}
\mathcal{V}^{\prime}= & f \frac{d \mathcal{V}}{d R} \\
= & -2 f\left[R^{2}+((1-a) P-3 \mu) R+(2 a-3) P \mu\right] \times \\
& \times\left(H^{-2 a-1} R^{-5}\right)=0,
\end{aligned}
$$

or, equivalently,

$R^{2}+((1-a) P-3 \mu) R+(2 a-3) P \mu=0$,

which yields the corresponding radius

$$
\begin{aligned}
R_{0} & =\frac{a-1}{2} P+\frac{3 \mu}{2}+\frac{1}{2} \sqrt{\mathcal{D}}, \\
\mathcal{D} & =(1-a)^{2} P^{2}+2(3-a) P \mu+9 \mu^{2}>0 .
\end{aligned}
$$

The inequality (72), which is valid for $0<a \leq 2$, is a trivial one.

It may be readily verified by using quadratic equation for $Z=R-2 \mu$

$Z^{2}+((1-a) P+\mu) Z-2 \mu^{2}-P \mu=0$

and $\mathcal{D}>0$ that

$R_{0}=R_{0,+}>2 \mu>R_{0,-}$,

for all $0<a \leq 2$. Here $R_{0,-}$ is another root of the quadratic equation (70), which corresponds to the "location" under the horizon and is irrelevant for our consideration.

The maximum of the eikonal part of the effective potential thus becomes

$\mathcal{V}_{0}=\mathcal{V}\left(R_{0}\right)=\frac{l(l+1)}{R_{0}^{2}}\left(1-\frac{2 \mu}{R_{0}}\right)\left(1+\frac{P}{R_{0}}\right)^{-2 a}$.

In Fig. 1 we plot the reduced eikonal part of the effective potential $\mathcal{V} /(l(l+1))(l \neq 0)$ as a function of the radial coordinate $R$ (left panel), and tortoise coordinate $R_{*}$ (right panel). As can be seen from examples presented in figure for special fixed values of $P$ and $\mu$, the maximum of the effective potential is largest for $a=0$ case and smallest for $a=2$ case. The case with $a=1$ is in the middle. At large distances the effective potential tends to zero, as expected.

The second derivative with respect to the tortoise coordinate is given by

$$
\begin{aligned}
\mathcal{V}_{0}^{\prime \prime}= & \left.\frac{d^{2} \mathcal{V}}{d R_{*}^{2}}\right|_{R_{*}=R_{*}\left(R_{0}\right)}=\left.f^{2} \frac{d^{2} \mathcal{V}}{d R^{2}}\right|_{R=R_{0}}=-\frac{2 l(l+1)}{R_{0}^{5}} \\
& \times\left(1-\frac{2 \mu}{R_{0}}\right)^{2}\left(1+\frac{P}{R_{0}}\right)^{-(1+4 a)} \sqrt{\mathcal{D}},
\end{aligned}
$$

where $\mathcal{D}$ is defined in (72).

The square of the cyclic frequency in the eikonal approximation reads as following $[12,13]$

$\omega^{2}=\mathcal{V}_{0}-i\left(n+\frac{1}{2}\right) \sqrt{-2 \mathcal{V}_{0}^{\prime \prime}}+O(1)$,

where $l \gg 1$ and $l \gg n$. Here $n=0,1, \ldots$ is the overtone number. By choosing an appropriate sign for $\omega$ we get the asymptotic relations (as $l \rightarrow+\infty$ ) on real and imaginary parts of complex $\omega$ in the eikonal approximation

$$
\begin{aligned}
\operatorname{Re}(\omega)= & \left(l+\frac{1}{2}\right) H_{0}^{-a} F_{0}^{1 / 2} R_{0}^{-1}+O\left(\frac{1}{l+\frac{1}{2}}\right), \\
\operatorname{Im}(\omega)= & -\left(n+\frac{1}{2}\right) H_{0}^{-a-1 / 2} F_{0}^{1 / 2} R_{0}^{-3 / 2} \mathcal{D}^{1 / 4} \\
& +O\left(\frac{1}{l+\frac{1}{2}}\right)
\end{aligned}
$$

where $H_{0}=1+\frac{P}{R_{0}}, F_{0}=1-\frac{2 \mu}{R_{0}}$ and $R_{0}, \mathcal{D}$ are given by (71), (72), respectively. 

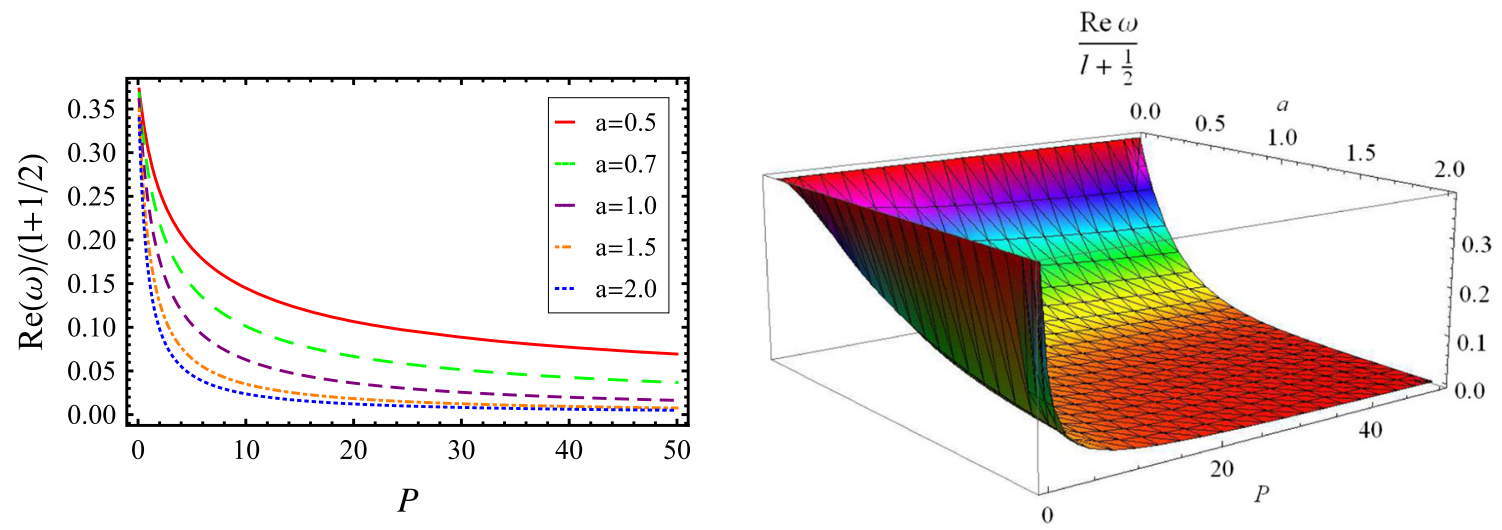

Fig. 2 The dependence (of eikonal limit) of $\operatorname{Re}(\omega) /(l+1 / 2)$ on $P$ for different $a$ in the range $0<a \leq 2$. Here we adopt $\mu=1 / 2$. Left panel: two dimensional plot. Right panel: three dimensional plot
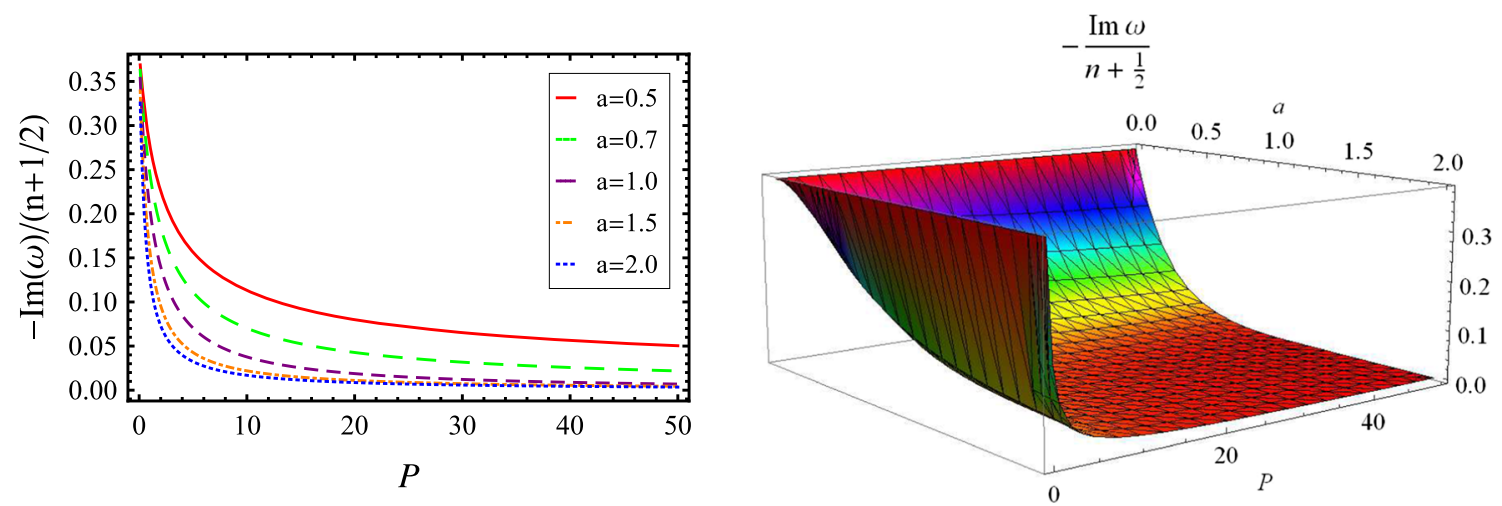

Fig. 3 The dependence (of eikonal limit) of $-\operatorname{Im}(\omega) /(n+1 / 2)$ on $P$ for different $a$ in the range $0<a \leq 2$. Here we adopt $\mu=1 / 2$. Left panel: two dimensional plot. Right panel: three dimensional plot

In Fig. 2 we constructed the (eikonal limit of) reduced real part of the QNM frequency, $\operatorname{Re}(\omega) /(l+1 / 2)$, as a function of parameter $P$ for different values of $a$ and $\mu=1 / 2$. In the right panel we have a three-dimensional plot of $\operatorname{Re}(\omega)$ versus $P$ and $a$. Here one can notice that in the limiting case when $a=0$ we recover constant $\operatorname{Re}(\omega)$ identical to the case of the Schwarzschild black hole.

In Fig. 3 we constructed the (eikonal limit of) reduced imaginary part of the QNM frequency with negative sign, $-\operatorname{Im}(\omega) /(n+1 / 2)$, as a function of parameter $P$ for different values of $a$ and $\mu=1 / 2$ in analogy to Fig. 2. At first sight Figs. 2 and 3 seem similar. However, according to Eqs. (78) and (79) this is not the case.

Remark It was shown in Ref. [41] that parameters of the unstable circular null geodesics around stationary spherically symmetric and asymptotically flat black holes are in correspondence with the eikonal part of quasinormal modes of these black holes. See also $[42,43]$ and references therein. But as it was pointed out in Ref. [44] this correspondence is valid if: (a) perturbations are described by a "good" effective potential, (b) "one is limited by perturbations of test fields only, and not of the gravitational field itself or other fields, which are non-minimally coupled to gravity." Here we do not consider this correspondence for our solution, postponing this to future publication.

\section{Limiting cases corresponding to the Schwarzschild and Reissner-Nordström black holes}

In this section we consider two limiting cases $a=+0$ and $a=2$ corresponding to the Schwarzschild and ReissnerNordström metrics, respectively.

(a) Let us first consider the case when $a=+0$. This limit may be obtained in a strong coupling regime when

$\lambda_{1}=\lambda e_{1}, \quad \lambda_{2}=\lambda e_{2}$,

where $e_{1}^{2}=e_{1}^{2}=1$, and

$e_{1} e_{2} \neq \pm 1, \quad \lambda \rightarrow+\infty$ 

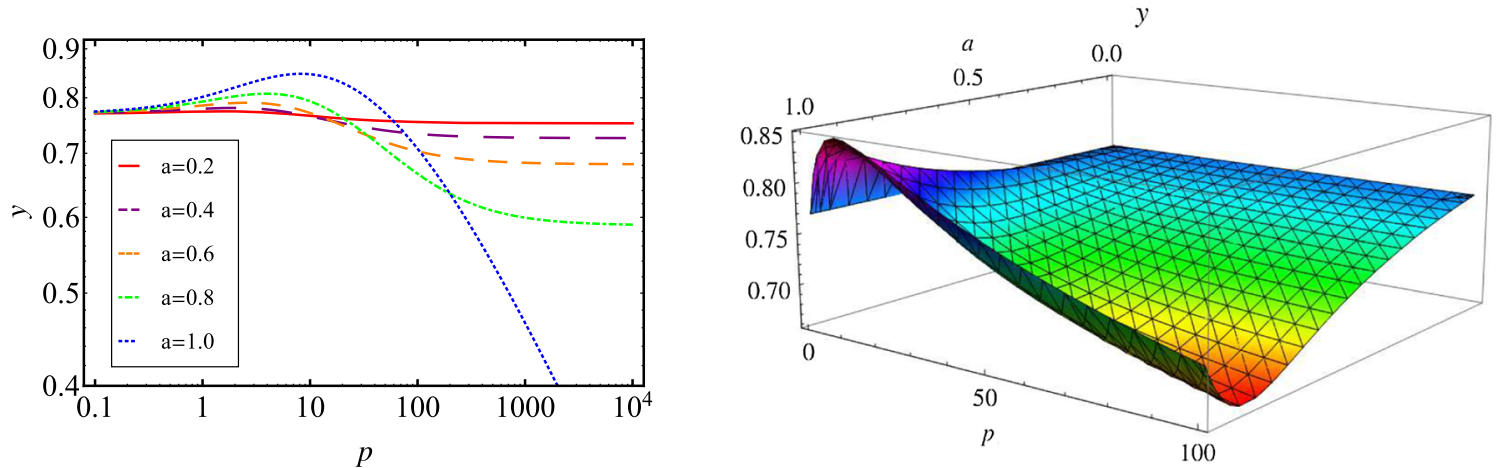

Fig. 4 The dependence of $y$ on $p$ for different $a$ (see (88)) in the range $0<a \leq 1$. Left panel: two dimensional plot in logarithmic scale. Right panel: three dimensional plot

Table 1 Maximum and limiting values of $y$ for various values of the model parameter $a$ in the ground state $n=0 . y_{\max }$ is the maximum of $y, p_{0}$ is the value of $p$ corresponding to $y_{\max }$ and $y_{\text {lim }}=\lim _{p \rightarrow \infty} y$

\begin{tabular}{llll}
\hline$a$ & $p_{0}$ & $y_{\max }$ & $y_{\text {lim }}$ \\
\hline $10^{-2}$ & 1.314 & 0.770 & 0.769 \\
0.1 & 1.421 & 0.772 & 0.762 \\
0.2 & 1.563 & 0.774 & 0.752 \\
0.4 & 1.954 & 0.780 & 0.726 \\
0.5 & 2.233 & 0.785 & 0.707 \\
0.6 & 2.608 & 0.790 & 0.681 \\
0.8 & 3.933 & 0.808 & 0.588 \\
1.0 & 8.196 & 0.847 & 0 \\
\hline
\end{tabular}

In this case the relations (78) and (79) for QNM in the eikonal approximation read as follows

$\operatorname{Re}(\omega)=\left(l+\frac{1}{2}\right) \sqrt{\frac{M}{r_{0}^{3}}}+O\left(\frac{1}{l+\frac{1}{2}}\right)$,

$\operatorname{Im}(\omega)=-\left(n+\frac{1}{2}\right) \sqrt{\frac{M}{r_{0}^{3}}}+O\left(\frac{1}{l+\frac{1}{2}}\right)$,

where $r_{0}=R_{0}=3 M$ corresponds the position where the black-hole effective potential attains its maximum. Note that $r_{0}=3 M$ is the radius of the photon sphere for the Schwarzschild black-hole. These results have been obtained in Ref. [7] and our outcomes are consistent with them.

(b) Now let us consider the case when $a=2$. As was mentioned above this takes place for collinear vectors $\lambda_{1}$, $\lambda_{2}$. One can also obtain the limit $a=2$ in the weak coupling regime when dilatonic coupling vectors obey (80) and

$e_{1} e_{2} \neq-1, \quad \lambda \rightarrow+0$.

In this case the eikonal QNM (see (78) and (79)) read

$\operatorname{Re}(\omega)=\left(l+\frac{1}{2}\right) \sqrt{\frac{M}{r_{0}^{3}}-\frac{Q^{2}}{2 r_{0}^{4}}}+O\left(\frac{1}{l+\frac{1}{2}}\right)$,
Table 2 Critical values of $p$ denoted as $p_{\text {crit }}$, obeying $y\left(p_{\text {crit }}\right)=1$, and $q_{\text {crit }}$ corresponding to $p_{\text {crit }}$ according to Eq. (94) for certain values of $a(1<a \leq 2)$

\begin{tabular}{lll}
\hline$a$ & $p_{\text {crit }}$ & $q_{\text {crit }}$ \\
\hline 1.1 & 8131.908 & 2.571 \\
1.2 & 18.157 & 2.274 \\
1.3 & 8.031 & 2.041 \\
1.4 & 5.402 & 1.870 \\
1.6 & 3.356 & 1.627 \\
1.8 & 2.461 & 1.457 \\
2.0 & 1.951 & 1.330 \\
\hline
\end{tabular}

$$
\begin{aligned}
\operatorname{Im}(\omega)= & -\left(n+\frac{1}{2}\right) \sqrt{\frac{M}{r_{0}^{3}}-\frac{Q^{2}}{2 r_{0}^{4}}} \sqrt{\frac{3 M}{r_{0}}-\frac{2 Q^{2}}{r_{0}^{2}}} \\
& +O\left(\frac{1}{l+\frac{1}{2}}\right),
\end{aligned}
$$

where $r_{0}=3 M / 2+(1 / 2) \sqrt{9 M^{2}-4 Q^{2}}=R_{0}+P$ corresponds to the position of the unstable, circular photon orbit in the Reissner-Nordström spacetime. These results have been obtained in Ref. [45] (for $n=0$ ) and our outcomes are compatible with them when the relation (for our notation) $Q^{2}=2 Q_{R N}^{2}$ is applied.

\section{Hod conjecture}

Here we test/check the conjecture formulated by Hod [40] on the existence of quasi-normal modes obeying the inequality

$|\operatorname{Im}(\omega)| \leq \pi T_{H}$,

where $T_{H}$ is the Hawking temperature.

Recently the Hod conjecture has been tested in theories with higher curvature corrections such as the 
Einstein-Dilaton-Gauss-Bonnet and Einstein-Weyl for the Dirac field [46]. It has been shown that in both theories the Dirac field obeys the Hod conjecture for the whole range of black-hole parameters [46].

Here we test/check this conjecture by using eikonal relations (79) for $\operatorname{Im}(\omega)$ and the relation for the Hawking temperature (37). For our purpose it is sufficient to check the validity of the inequality

$$
\begin{aligned}
y \equiv & \frac{\left|\operatorname{Im}\left(\omega_{e i k}\right)(n=0)\right|}{\pi T_{H}}=4\left(1+\frac{p}{x}\right)^{-a-1 / 2} \\
& \times\left(1-\frac{2}{x}\right)^{1 / 2} x^{-3 / 2} d^{1 / 4}\left(1+\frac{p}{2}\right)^{a}<1,
\end{aligned}
$$

for all $p=P / \mu>0$, where

$x \equiv R_{0} / \mu=\frac{a-1}{2} p+\frac{3}{2}+\frac{1}{2} \sqrt{d}$,

$d \equiv \mathcal{D} / \mu^{2}=(1-a)^{2} p^{2}+2(3-a) p+9>0$.

In (88) we use the limiting "eikonal value" given by the first term in (79) for the lowest overtone number $n=0$.

In Table 1 we present the results for the numerical testing of the Hod bound by using obtained relations for the eikonal QNM in the ground state $n=0$. It turned out that the Hod bound is valid (in the eikonal regime) in the range $0<a \leq 1$. There are maximum $y_{\max }=y_{\max }(a)$ and limiting $y_{\text {lim }}=y_{\text {lim }}(a)$ values of function $y(p, a)$ for different values of parameter $a$ in the considered range.

It may be verified that

$y_{\text {lim }}(a)=\left(\frac{1-a}{3-2 a}\right)^{3 / 2-a} 2^{2-a}<\frac{4}{3 \sqrt{3}}<1$

for $0<a<1$ and $y_{\text {lim }}(1)=0$. The relation for $y_{\text {lim }}(a)$ just follows from

$$
\lim _{p \rightarrow+\infty} x(p, a)=(3-2 a) /(1-a)
$$

for $0<a<1$.

We denote the value of $p$ corresponding to $y_{\max }(a)$ as $p_{0}=p_{0}(a)$. For increasing $a$, the values of $p_{0}(a)$ and $y_{\max }(a)$ increase and $y_{\text {lim }}(a)$ decrease. We obtain $y_{\max }(1) \approx$ 0.847 and $y_{\text {lim }}(+0)=0$. For decreasing $a$, both $y_{\max }(a)$ and $y_{\text {lim }}(a)$ approach a finite value, corresponding to the Schwarzschild case $\frac{4}{3 \sqrt{3}} \approx 0.7698$ when $a \rightarrow 0$.

In Fig. 4 we illustrate $y=y(p, a)$ as a function of $p$. In the left panel we build two dimensional plot for $a=0.2,0.4,0.6$, $0.8,1.0$ and in the right panel we construct three dimensional plot for the range $0<a \leq 1$, where the Hod conjecture holds. Thus, we are led to the following proposition.

Proposition The dimensionless parameter $y=y(p, a)$ from (88) obeys the inequality: $y(p, a)<1$ for all $p>0$ and $a \in(0,1]$.
For $0<a<1$ this proposition is proved analytically in Appendix. For $a=1$ it is justified by our numerical bound $y<y_{\max }(1) \approx 0.847$.

In addition we considered the range $1<a \leq 2$ for testing the Hod conjecture. In this case we have

$y_{\text {lim }}(a)=\lim _{p \rightarrow+\infty} y(p, a)=+\infty$

due to asymptotic relation $y(p, a) \sim C(a) p^{a-1}$ for $p \rightarrow$ $+\infty$ following from $x(p, a) \sim(a-1) p$, where $C(a)=$ $2^{2-a}(a /(a-1))^{-a-1 / 2}(a-1)^{-1}$. Strictly speaking for $1<$ $a \leq 2$ there exist critical values $p_{\text {crit }}(a)$ of parameter $p$ such that for $p \in\left(0, p_{\text {crit }}(a)\right)$ the Hod conjecture holds in eikonal regime while for $p \in\left(p_{\text {crit }}(a),+\infty\right)$ it fails (see Fig. 5 for details). Here the limit $p=+\infty$ corresponds to extremal (black hole) case which is not considered here.

Remark Recently, in Ref. [47] some example of the violation of the Hod conjecture has been found for certain (scalar gravitational) perturbations around $D=5$ Gauss-Bonnet-de Sitter black hole solution.

In Table 2 we present some critical values $p_{\text {crit }}$, which are obtained through the condition $y\left(p_{\text {crit }}\right)=1$ (with $y$ calculated for the ground state $n=0$ ) and $q_{\text {crit }}$ corresponding to $p_{\text {crit }}$ according to Eq. (94) for various values of the model parameter $a$ obeying $1<a \leq 2$.

Here we use the following relation (see Eq. (32))

$q=\frac{|Q|}{M}=\frac{2 \sqrt{2} \sqrt{p(2+p)}}{2+a p}<\frac{2 \sqrt{2}}{a}=q_{\text {ext }}$,

where $q_{\text {ext }}$ corresponds to extremal case. (Here $G=1$.)

In Fig. 5 we illustrate $y=y(p, a)$ as a function of $p$. In the left panel we build two dimensional plot for $a=1.2$, $1.3,1.4,1.6,2.0$ and in the right panel we construct three dimensional plot for the range $1<a \leq 2$. In this case the Hod inequality (88) holds in the range $p \in\left(0, p_{\text {crit }}(a)\right)$, while for $p \in\left(p_{\text {crit }}(a),+\infty\right)$ it breaks.

Remark In Ref. [48], the eikonal QNM frequencies for charged scalar field in the space-time of a charged ReissnerNordström black hole were obtained analytically in the regime $l^{2} \geq Q q_{*} \geq l$, where $Q$ is the electric charge of the black hole and $q_{*}$ is the electric charge of the field. In this regime the obtained fundamental frequencies were shown to saturate the Hod bound. It should be noted that this result can not be applied to our analysis for $a=2$, since we deal here with $q_{*}=0$ case.

\section{Conclusions}

We have examined a non-extremal black hole dyon-like solution in a 4-dimensional gravitational model with two scalar 

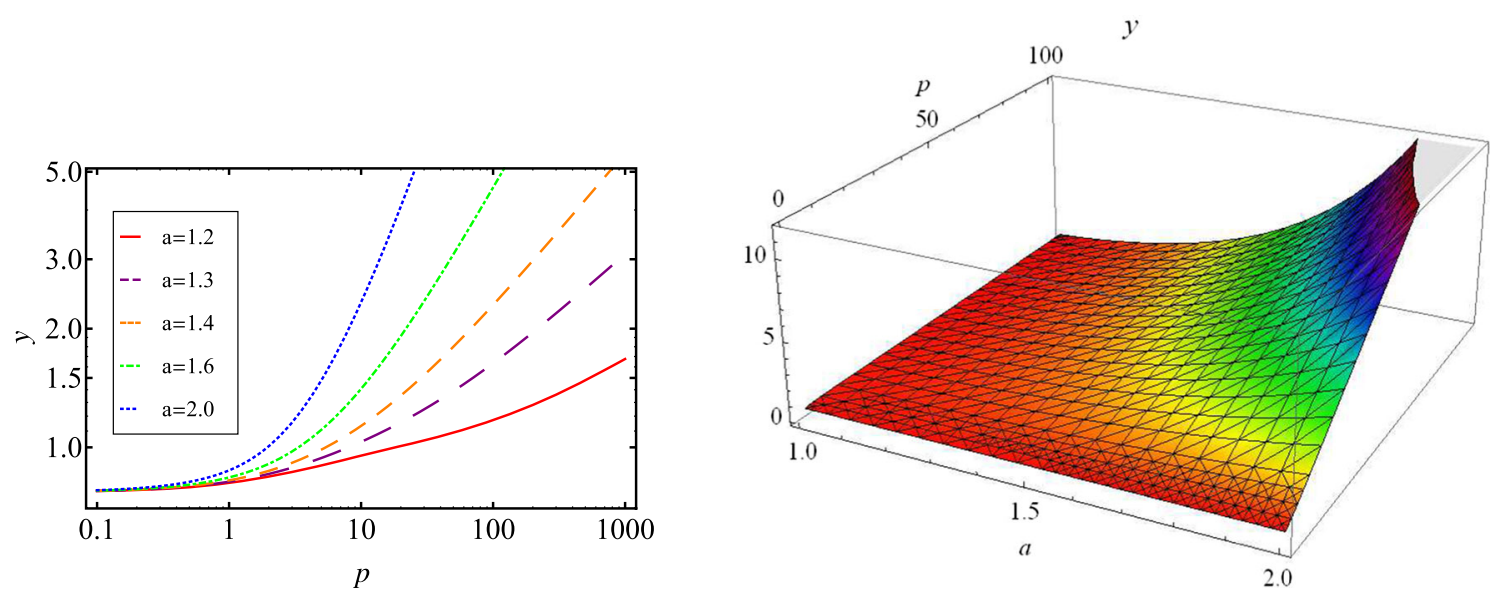

Fig. 5 The dependence of $y$ on $p$ for different $a$ (see (88) in the range $1<a \leq 2$. Left panel: two dimensional plot in logarithmic scale. Right panel: three dimensional plot

fields and two Abelian vector fields proposed in Ref. [30]. The model contains two vectors of dilatonic coupling vectors $\lambda_{s} \neq 0, s=1,2$, obeying $\lambda_{1} \neq-\lambda_{2}$ and additional relations (18). We have also presented some physical parameters of the solutions: gravitational mass $M$, scalar charges $Q_{\varphi}^{i}$, Hawking temperature, black hole area entropy. In addition, we considered the first law of black hole thermodynamics and checked the validity of the Smarr relation for our model.

In fact this is a special solution with dependent electric and magnetic charges, see (17). In the case of non-collinear vectors $\lambda_{1}, \lambda_{2}$ the metric of the solution describes a black hole with one (external horizon) and singularity hidden by it. For collinear vectors $\lambda_{1}, \lambda_{2}$ the metric coincides with the Reissner-Nordström metric possessing two horizons and hidden singularity.

We have studied the solutions to massless (covariant) Klein-Fock-Gordon equation in the background of our static and spherically symmetric metric, by using the variable separation method. The Klein-Fock-Gordon equation is simplified in the tortoise coordinate leading to radial equation governed by effective potential. This potential contains the parameters of solution such as $P>0, \mu>0$ and dimensionless parameter $a \in(0,2]$ depending upon the coupling vectors $\lambda_{s}$ which are the initial parameters of the model. The physical quantities, such as mass, color charges and scalar charges contain some of these parameters.

Here we mainly focused on the eikonal part of the effective potential and calculated the value of the radial coordinate (radius) $R_{0}$ corresponding to the maximum of this part of the effective potential. Knowing the maximum of the eikonal part of the effective potential and corresponding radius, we have calculated the cyclic frequencies of the quasinormal modes in the eikonal approximation. We have also considered two limiting cases reducing to the Schwarzschild and ReissnerNordström solutions, when the parameter of the solution $a$ accepts two distinct values, i.e. $a=+0$ and $a=2$, respectively. Thus, we have made sure that our outcomes are consistent with the previous results in the literature.

We have also tested the validity of the Hod conjecture for our solution by using QNM frequencies in the eikonal approximation with the lowest value of the overtone number $n=0$. It turned out that the Hod assumption holds in the range of $0<a \leq 1$. The conjecture is valid in this range since it is supported by examples of states with large enough values of angular number $l$. For $1<a \leq 2$ we have found that the Hod bound is satisfied for $n=0$, and small enough values of charge $Q\left(Q / M<q_{\text {crit }}(a)\right)$ and big enough values of $l(l \gg 1)$.

It would be interesting to explore in detail QNM frequencies in the vicinity of $a=0$ and $a=2$, by using the treatment of Ref. [49], e.g. extending the results for the

Schwarzschild and Reissner-Nordström solutions by using expansion in a small parameter ( $a$ or $a-2)$. Another issue of interest is the numerical calculation of QNM frequencies by using higher-order WKB formula for certain lower levels (labelled by $l$ and $n$ ), see Ref. [50], e.g. verifying the Hod conjecture for $1<a<2$, calculating grey-body factors etc. All these issues may be addressed in our future studies.

Acknowledgements This paper has been supported by the RUDN University Strategic Academic Leadership Program (recipient: V.D.I. - mathematical model development) and by the Ministry of Education and Science of the Republic of Kazakhstan, project identification registration number (IRN): AP08052311 (recipients K.B. - simulation model development and numerical checking of results). The authors thank Roman Konoplya for fruitful discussions and valuable comments during the preparation of the manuscript.

Data Availability Statement This manuscript has no associated data or the data will not be deposited. [Authors' comment: All data generated or analysed during this study are included in this published article.] 
Open Access This article is licensed under a Creative Commons Attribution 4.0 International License, which permits use, sharing, adaptation, distribution and reproduction in any medium or format, as long as you give appropriate credit to the original author(s) and the source, provide a link to the Creative Commons licence, and indicate if changes were made. The images or other third party material in this article are included in the article's Creative Commons licence, unless indicated otherwise in a credit line to the material. If material is not included in the article's Creative Commons licence and your intended use is not permitted by statutory regulation or exceeds the permitted use, you will need to obtain permission directly from the copyright holder. To view a copy of this licence, visit http://creativecomm ons.org/licenses/by/4.0/.

Funded by SCOAP ${ }^{3}$.

\section{Appendix A: Analytic proof of the Hod conjecture}

Here we prove the Proposition from Sect. 6 for $0<a<1$. The quadratic equation for $x=x(p, a)$ from (89) in terms of $a, p$ reads

$\left.x^{2}-[(a-1) p+3)\right] x+(2 a-3) p=0$,

see (70). Due to (74) we have $x>2$. Substituting $x=u+2$, we get

$u^{2}+[(1-a) p+1] u=2+p=1+\frac{t-a}{1-a}$,

where $t=1+(1-a) p, t>1$ and $u>0$ (since $u=u_{+}$is the large root of the quadratic equation (A.2) and the small root $u=u_{-}$obeys $\left.u_{+} u_{-}=-2-p<0\right)$. See also Eq. (73) for $Z=u \mu(\mu>0)$.

Thus,

$p / 2+1=u(u+t) / 2$,

see (A.2). Next, from the Eq. (A.2) we get

$[1-(1-a) u] p=(u-1)(u+2)$,

which implies (due to $p>0, u>0$ )

$1<u<(1-a)^{-1}$,

and hence

$p=\frac{(u-1)(u+2)}{1-(1-a) u}$,

so

$\frac{p}{x}+1=\frac{a u}{1-(1-a) u}$.

Finally, from the equation for $x$ (89), we get

$\sqrt{d}=2 x-(a-1) p-3=2 u+t$.

Plugging all of that in (88), we see that we need to prove the following bound

$y=4\left[\frac{(u+t)(1-(1-a) u)}{2 a}\right]^{a}\left[\frac{1-(1-a) u}{a u}\right]^{1 / 2}$

$$
\times\left[\frac{u}{u+2}\right]^{1 / 2} \frac{(t+2 u)^{1 / 2}}{(u+2)^{3 / 2}}<1 .
$$

Now, from (A.2)

$(1-a) u(t+u)=1+t-2 a$,

so the first bracket in (A.9) simplifies to

$$
\begin{aligned}
\left(\frac{u-1+2 a}{2 a}\right)^{a} & =\left(1+\frac{u-1}{2 a}\right)^{a} \\
& \leq 1+\frac{u-1}{2}=\frac{u+1}{2} .
\end{aligned}
$$

Here we have used well-known convexity inequality $(1+$ $v)^{a} \leq 1+a v$ which is valid for $v>-1$ and $0<a \leq 1$ (for our case $v>-1$ is valid due to (A.5)). We can cancel $u^{1 / 2}$ and combine all $(u+2)$ 's, which leaves us with the factor

$F(t)=\frac{(1-(1-a) u)(2 u+t)}{a}$.

Here we use the differentiation tool

$\frac{\mathrm{d}}{\mathrm{dt}} \ln F(t)=-\frac{(1-a) \dot{u}}{1-(1-a) u}+\frac{2 \dot{u}+1}{2 u+t}$,

where $\dot{u}=d u / d t$. From (A.2), we have

$(1-a)(2 u+t) \dot{u}=1-(1-a) u$,

and hence $\dot{u}>0$ (see (A.5)). Using (A.14) we obtain

$\frac{\mathrm{d}}{\mathrm{d} t} \ln F(t)=-\frac{1}{2 u+t}+\frac{2 \dot{u}+1}{2 u+t}=\frac{2 \dot{u}}{2 u+t}$,

so the logarithmic derivative evaluates to

$\frac{\mathrm{d}}{\mathrm{d} t} \ln F(t)=\frac{2 \dot{u}}{2 u+t}<\frac{2 \dot{u}}{2 u+1}=\frac{\mathrm{d}}{\mathrm{d} t} \ln (2 u+1)$

(here we use $\dot{u}>0$ and $t>1$ ). Thus, integrating (A.16) (in $t$ from 1 to $t$ ) and using coincidence of initial values: $F(1)=2 u(1)+1=3(u(t) \rightarrow 1$ as $t \rightarrow 1$ and $p \rightarrow+0)$ we get

$F(t)<2 u+1$

and we end up with proving that

$2(u+1) \sqrt{2 u+1}<(u+2)^{2}$.

Indeed, $(u+2)^{2}-2(u+1) \sqrt{2 u+1}$

$=2+(u+1-\sqrt{2 u+1})^{2}>0$. This ends the proof of the inequality (A.9), or, equivalently, $y(p, a)<1$ for all positive $p$ and $0<a<1$.

\section{References}

1. B.P. Abbott et al., Phys. Rev. Lett. 116(6), 061102 (2016). https:// doi.org/10.1103/PhysRevLett.116.061102

2. B.P. Abbott et al., Phys. Rev. X 9(3), 031040 (2019). https://doi. org/10.1103/PhysRevX.9.031040 
3. B.P. Abbott et al., Astrophys. J. Lett. 892(1), L3 (2020). https:// doi.org/10.3847/2041-8213/ab75f5

4. C.V. Vishveshwara, Nature 227(5261), 936 (1970). https://doi.org/ 10.1038/227936a0

5. W.H. Press, Astrophys. J. Lett. 170, L105 (1971). https://doi.org/ $10.1086 / 180849$

6. S. Chandrasekhar, S. Detweiler, Proc. R. Soc. Lond. Ser. A 344(1639), 441 (1975). https://doi.org/10.1098/rspa.1975.0112

7. H.J. Blome, B. Mashhoon, Phys. Lett. A 100(5), 231 (1984). https:// doi.org/10.1016/0375-9601(84)90769-2

8. V. Ferrari, B. Mashhoon, Phys. Rev. Lett. 52(16), 1361 (1984). https://doi.org/10.1103/PhysRevLett.52.1361

9. V. Ferrari, B. Mashhoon, Phys. Rev. D 30(2), 295 (1984). https:// doi.org/10.1103/PhysRevD.30.295

10. K.D. Kokkotas, B.G. Schmidt, Living Rev. Relativ. 2(1), 2 (1999). https://doi.org/10.12942/lrr-1999-2

11. H.P. Nollert, Class. Quantum Gravity 16(12), R159 (1999). https:// doi.org/10.1088/0264-9381/16/12/201

12. E. Berti, V. Cardoso, A.O. Starinets, Class. Quantum Gravity 26(16), 163001 (2009). https://doi.org/10.1088/0264-9381/26/16/ 163001

13. R.A. Konoplya, A. Zhidenko, Rev. Mod. Phys. 83(3), 793 (2011). https://doi.org/10.1103/RevModPhys.83.793

14. Y. Hatsuda, Phys. Rev. D 101(2), 024008 (2020). https://doi.org/ 10.1103/PhysRevD.101.024008

15. J.C. Fabris, M.G. Richarte, A. Saa, Phys. Rev. D 103(4), 045001 (2021). https://doi.org/10.1103/PhysRevD.103.045001

16. M.E. Abishev, K.A. Boshkayev, V.D. Dzhunushaliev, V.D. Ivashchuk, Class. Quantum Gravity 32(16), 165010 (2015). https:// doi.org/10.1088/0264-9381/32/16/165010

17. M.E. Abishev, K.A. Boshkayev, V.D. Ivashchuk, Eur. Phys. J. C 77(3), 180 (2017). https://doi.org/10.1140/epjc/ s10052-017-4749-1

18. M.E. Abishev, V.D. Ivashchuk, A.N. Malybayev, S. Toktarbay, Gravit. Cosmol. 25(4), 374 (2019). https://doi.org/10.1134/ S0202289319040029

19. K. Bronnikov, G. Shikin, Russ. Phys. J. 20, 1138 (1977)

20. G.W. Gibbons, Nucl. Phys. B 207(2), 337 (1982). https://doi.org/ 10.1016/0550-3213(82)90170-5

21. G.W. Gibbons, K.I. Maeda, Nucl. Phys. B 298(4), 741 (1988). https://doi.org/10.1016/0550-3213(88)90006-5

22. D. Garfinkle, G.T. Horowitz, A. Strominger, Phys. Rev. D 43(10), 3140 (1991). https://doi.org/10.1103/PhysRevD.43.3140

23. G.J. Cheng, R.R. Hsu, W.F. Lin, J. Math. Phys. 1 35, 4839 (1994)

24. G.W. Gibbons, D. Kastor, L. London, P. Townsend, J. Traschen, Nucl. Phys. B 416, 850 (1994)

25. S.J. Poletti, J. Twamley, D.L. Wiltshire, Class. Quantum Gravity 12(7), 1753 (1995). https://doi.org/10.1088/0264-9381/12/7/017

26. S.B. Fadeev, V.D. Ivashchuk, V.N. Melnikov, L.G. Sinanyan, Gravit. Cosmol. 7, 343 (2001)

27. D. Gal'tsov, M. Khramtsov, D. Orlov, Phys. Lett. B 743, 87 (2015). https://doi.org/10.1016/j.physletb.2015.02.017

28. E.A. Davydov, Theor. Math. Phys. 197(2), 1663 (2018). https:// doi.org/10.1134/S0040577918110107
29. A. Zadora, D.V. Gal'tsov, C.M. Chen, Phys. Lett. B 779, 249 (2018). https://doi.org/10.1016/j.physletb.2018.02.017

30. F.B. Belissarova, K.A. Boshkayev, V.D. Ivashchuk, A.N. Malybayev, J. Phys.: Conf. Ser. 1690, 012143 (2020). https://doi.org/ 10.1088/1742-6596/1690/1/012143

31. R.A. Konoplya, Gen. Relativ. Gravit. 34, 329 (2002). https://doi. org/10.1023/A:1015347628961

32. V. Ferrari, M. Pauri, F. Piazza, Phys. Rev. D 63(6), 064009 (2001). https://doi.org/10.1103/PhysRevD.63.064009

33. R.A. Konoplya, Phys. Rev. D 66(8), 084007 (2002). https://doi. org/10.1103/PhysRevD.66.084007

34. S. Chen, J. Jing, Class. Quantum Gravity 22(6), 1129 (2005). https://doi.org/10.1088/0264-9381/22/6/014

35. H. Nomura, T. Tamaki, J. Phys. Conf. Ser. 24, 123-129 (2005). https://doi.org/10.1088/1742-6596/24/1/014

36. I. Sakalli, Mod. Phys. Lett. A 28(27), 1350109 (2013). https://doi. org/10.1142/S0217732313501095

37. K.D. Kokkotas, R.A. Konoplya, A. Zhidenko, Phys. Rev. D 92(6), 064022 (2015). https://doi.org/10.1103/PhysRevD.92.064022

38. C. Pacilio, R. Brito, Phys. Rev. D 98(10), 104042 (2018). https:// doi.org/10.1103/PhysRevD.98.104042

39. J.L. Blázquez-Salcedo, S. Kahlen, J. Kunz, Eur. Phys. J. C 79(12), 1021 (2019). https://doi.org/10.1140/epjc/s10052-019-7535-4

40. S. Hod, Phys. Rev. D 75(6), 064013 (2007). https://doi.org/10. 1103/PhysRevD.75.064013

41. V. Cardoso, A.S. Miranda, E. Berti, H. Witek, V.T. Zanchin, Phys. Rev. D 79(6), 064016 (2009). https://doi.org/10.1103/PhysRevD. 79.064016

42. K.S. Virbhadra, G.F.R. Ellis, Phys. Rev. D 62(8), 084003 (2000). https://doi.org/10.1103/PhysRevD.62.084003

43. M. Cvetič, G.W. Gibbons, C.N. Pope, Phys. Rev. D 94(10), 106005 (2016). https://doi.org/10.1103/PhysRevD.94.106005

44. R.A. Konoplya, Z. Stuchlík, Phys. Lett. B 771, 597 (2017). https:// doi.org/10.1016/j.physletb.2017.06.015

45. N. Andersson, H. Onozawa, Phys. Rev. D 54(12), 7470 (1996). https://doi.org/10.1103/PhysRevD.54.7470

46. A.F. Zinhailo, Eur. Phys. J. C 79(11), 912 (2019). https://doi.org/ 10.1140/epjc/s10052-019-7425-9

47. M.A. Cuyubamba, R.A. Konoplya, A. Zhidenko, Phys. Rev. D 93(10), 104053 (2016). https://doi.org/10.1103/PhysRevD.93. 104053

48. S. Hod, Phys. Lett. B 710(2), 349 (2012). https://doi.org/10.1016/ j.physletb.2012.03.010

49. M.S. Churilova, Eur. Phys. J. C 79(7), 629 (2019). https://doi.org/ 10.1140/epjc/s10052-019-7146-0

50. R.A. Konoplya, A. Zhidenko, A.F. Zinhailo, Class. Quantum Gravity 36(15), 155002 (2019). https://doi.org/10.1088/1361-6382/ ab2e25 